\title{
Is Ki-67 of Diagnostic Value in Distinguishing Between Partial and Complete Hydatidiform Moles? A Systematic Review and Meta-analysis
}

\author{
YUE ZHAO $^{1,2}$, GUANG-WU XIONG ${ }^{3}$, XIAO-WEI ZHANG ${ }^{1}$ and BO HANG ${ }^{2}$ \\ ${ }^{1}$ Department of Obstetrics and Gynecology, Peking University Third Hospital, Beijing, P.R. China; \\ ${ }^{2}$ Biological Sciences \& Engineering Division, Lawrence Berkeley National Laboratory, \\ University of California, Berkeley, CA, U.S.A.; \\ ${ }^{3}$ Women \& Children Health Center, The Third Affiliated Hospital of Chongqing Medical University, \\ Chongqing, P.R. China
}

\begin{abstract}
Background/Aim: To demonstrate the value of Ki-67 in distinguishing between partial and complete hydatidiform moles. Materials and Methods: We searched electronic databases included Medline, WOK, Cochrane Library and CNKI, through January 24, 2015. Experts were consulted, and references from related articles were examined. The meta-analysis was conducted with RevMan5.3, according to the PRISMA guidelines. MantelHaenszel estimates were calculated and pooled under a random effect model, with data expressed as odds ratio (OR) and 95\% confidence interval (CI). Results: We analyzed eight trials with a total of 337 participants who underwent uterine curettage and met the inclusion criteria. A significantly higher expression of Ki-67 was observed in complete than in partial hydatidiform moles $(\mathrm{OR}=3.28$; 95\%CI=1.80-5.96; $p<0.0001)$. Conclusion: The Ki-67 expression was higher in complete than in partial hydatidiform moles. Therefore, Ki-67 may be of diagnostic value in distinguishing between partial and complete hydatidiform moles. However, the present study had only a limited number of samples, so investigation of a greater number of cases is needed to confirm this conclusion.
\end{abstract}

Correspondence to: Xiao-Wei Zhang, Department of Obstetrics and Gynecology, Peking University Third Hospital, 49 North Garden Rd., Haidian District Beijing 100083, P.R. China. Tel: +86 13811535057, e-mail: 1411110301@bjmu.edu.cn; Bo Hang, Lawrence Berkeley National Laboratory, MS1-325, One Cyclotron Road, University of California, Berkeley, CA 94720, U.S.A. Tel: +1 5109937377, e-mail: 7456216@gmail.com

Key Words: Diagnosis, hydatidiform mole, Ki-67 antigen, metaanalysis.
The hydatidiform mole encompasses a heterogeneous group of lesions that feature an abnormal non-neoplastic proliferation of trophoblasts (1). This mole can be classified into two subsidiary sets based on clinical, morphological and genetic characteristics (2). The complete hydatidiform mole is a diploid androgenetic conceptus with indiscriminate hyperplasia of the villous trophoblast and generalized swelling of the hydatidiform villous without detectable fetal tissues. The partial hydatidiform mole is characterized by a diandric triploid conceptus, accompanying focal trophoblastic hyperplasia and villous hydrops, and an ascertainable fetus. Clinically, complete moles show a much higher incidence (18-29\%) of developing into gestational trophoblastic neoplasia, whereas persistent trophoblastic neoplasia occurs only in $1.0-5.6 \%$ of partial mole cases (3, $4)$. Therefore, the possibility of progressing to gestational trophoblastic neoplasia and the need for clinical processing and follow-up of the patient emphasizes the importance of differentiating between complete and partial hydatidiform moles. However, this is rather difficult and the two mole types are easily mistaken (5).

Resulting from the earlier clinical evaluation and management of hydatidiform moles, the histopathological features which were regarded as diagnostic evidence of distinguishing complete moles from partial moles are more subtle and less readily identifiable $(3,6)$. In addition, interobserver and intraobserver variability reduces the efficiency of the previously well-described histopathologic criteria $(7,8)$. During routine clinical practice, misdiagnosis of complete and partial moles is not a new story $(9,10)$. On the other hand, the recognition that the different subsidiary sets have different clinical management and prognosis has made the precise subdivision of hydatidiform moles essential.

At present, many detection methods have been developed (e.g. DNA ploidy analysis, short-tandem-repeat (STR) 
genotyping and chromosomal enumeration by fluorescent in situ hybridisation (FISH)) (11-13); most of these are based at the genetic level (14) and are too time-consuming, inconvenient and economically inefficient for the clinical setting. Thus, a fairly rapid, simple and economical technique is needed to serve as a useful adjunct to conventional methods in the diagnosis of gestational trophoblastic diseases. In this context, several different biomarkers targeting the subclassification of hydatidiform moles have been explored to improve diagnostic accuracy.

Healthy trophoblasts and malignant cells share many similarities, such as invasiveness, high cell proliferation, lack of cell contact inhibition, and immune privilege. Quantitative assessment of immunoreactivity of Ki-67 antigen (MIB1) through the use of immunohistochemical methods has been established as a valuable reflection of cell proliferative potential in cancer, and could, therefore, be of value in studying the biological behavior of molar gestations (15-20). Immunohistochemical methods are a relatively simple alternative to other more complex genetic techniques. One of the advantages of immunohistochemical methods is the ability to apply them retrospectively to sections of routinely formalin-fixed and paraffin-embedded tissue. Another advantage is that, unlike the case for deoxyribonucleic acid ploidy analysis techniques, no expensive or sophisticated equipment is needed.

However, the use of $\mathrm{Ki}-67$ suffers from a lack of consensus regarding its diagnostic value. We addressed this significant and controversial question by performing a systematic review and meta-analysis in order to assess the potential value of Ki-67 in distinguishing between partial and complete hydatidiform moles and for making a precise subdivision between them. The overall goal was to determine whether measuring the immunoreactivity of the Ki-67 antigen using immunohistochemical methods might improve the accuracy of differential diagnosis of gestational trophoblastic disease subgroups.

\section{Materials and Methods}

Systematic search strategy. We conducted a comprehensive search of electronic databases, including Medline, WOK, Cochrane Library and CNKI, through January 24, 2015, to identify all relevant studies. Experts were consulted, and references from relevant articles were scanned. The detailed search strategy for Medline with Medical Subject Heading search terms and free-text related to Ki-67 and gestational trophoblastic diseases can be found in Table I. Studies were considered regardless of language status. Two independent reviewers performed all aspects, including the search strategy, screening the titles and abstracts of all articles according to inclusion criteria and then reviewing the full-text articles in detail, as indicated. The third reviewer examined and arbitrated any disagreements.

Identification of articles and data extractions. Trials meeting all the following terms were eligible for inclusion: (1) trials assessing the
Table I. Detailed search strategy for Medline.

\begin{tabular}{ll}
\hline NO. & Search Terms \\
\hline$\# 1$ & "hydatidiform moles" (MeSH) \\
$\# 2$ & "vesicular mole" \\
$\# 3$ & "cystic mole" \\
$\# 4$ & "hydatid mole" \\
$\# 5$ & "mole" \\
$\# 6$ & \#1 OR \#2 OR \#3 OR \#4 OR \#5 \\
$\# 7$ & "Ki-67" \\
$\# 8$ & "MKI67" \\
$\# 9$ & "KIA" \\
$\# 10$ & "MIB-1" \\
$\# 11$ & $\# 7$ OR \#8 OR \#9 OR \#10 \\
$\# 12$ & \#6 AND \#11 \\
\hline
\end{tabular}

MeSH: Medical subject heading.

pathologic specimen which were obtained from uterine curettage and with definite diagnosis of hydatidiform moles; (2) trials performed with immunohistochemical techniques to compare the immunoreactivity of Ki67 antigen between partial and complete hydatidiform moles; (3) trials with explicit specification of sample content and related comprehensive statistics; (4) trials with quantitative (percentage of positive cells) or semi-quantitative assessment of immunoreactivity of Ki67 antigen (to be more specific, $(-)$ with less than $5 \%$ cells stained, $(+) 5-25 \%$ of cells stained, $(++) 26-50 \%$ of cells stained and $(+++)>50 \%$ of cells stained); (5) for repeated reports of the same sample population, only the most recently published one included.

A standardized table was used to extract the required data from all eligible publications including study details (first author's name, publication year, and country), participant details (number of patients in respective groups), and results (distribution of Ki-67 immunoreactivity).

The Newcastle-Ottawa Scale (NOS) was used to assess the quality of the included studies independently by two reviewers (21). The two investigators extracted data until an agreement was reached through discussion.

Data synthesis and data analysis. Dichotomous data were presented as odds ratios (ORs) with 95\% confidence intervals (CIs) to compare the expression of $\mathrm{Ki}-67$ in partial and complete hydatidiform moles. The Mantel-Haenszel estimates were calculated and pooled under a random effects model. Heterogeneity was assessed by examining the clinical characteristics of the included studies as well as by formal statistical testing with $\chi^{2}$ and $\mathrm{I}^{2}$. The Egger test was used to assess the possibility of publication bias by Stata/MP 13.0 (StataCorp, College Station, TX, USA). Metaanalysis was performed with RevMan5.3 according to the PRISMA guidelines (22).

Studies selection and characteristics. The systematic search identified 317 relevant references (Figure 1). After screening titles and abstracts, we excluded 274 articles, such as those lacking subdivision of hydatidiform moles and repeated trials. The 43 remaining articles were retrieved in full text for formal review. After independent review, 35 studies failing to offer quantitative or semi- 


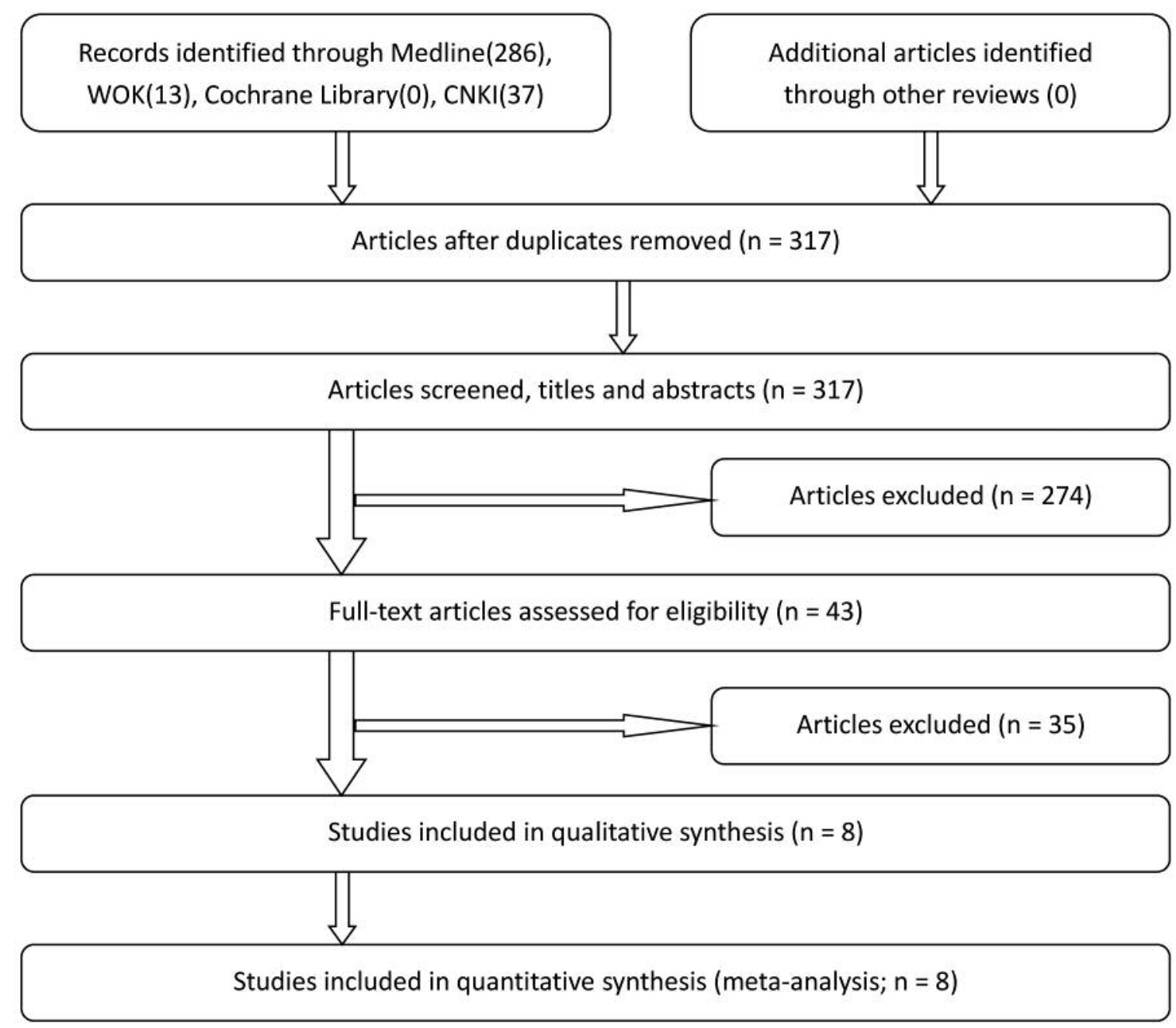

Figure 1. Search strategy to identify trials for the expression of Ki-67 in complete and partial hydatidiform moles. WOK: Web of Knowledge.

quantitative data and intermediate analysis were excluded. We examined recent reviews and comments, and no additional relevant trial was included.

Table II displays the characteristics of the eight identified studies containing 337 participants that met our predefined inclusion criteria (23-30). Patient characteristics, such as the number of participants in each study, were collected and Ki-67 levels were also gathered.

\section{Results}

Eight trials were analyzed, with a total of 337 participants who suffered hydatidiform moles and met the inclusion criteria. Positivity for Ki-67 was found in 163 of the 188 cases with complete hydatidiform moles, compared with 103 of the 149 cases with partial hydatidiform moles, resulting in an OR of 3.28 (95\% CI=1.80-5.96; $p<0.0001$; Figure 2). Little heterogeneity was noted among these trials $\left(\mathrm{I}^{2}=0 \%\right.$; $\left.\chi^{2}=1.74 ; p=0.88\right)$. Egger's test and the funnel plots showed little publication bias for the overall analysis (Figure 3 ).

\section{Discussion}

The constant enrichment and modification of diagnosis technology in recent years has significantly improved the early detection rate and prognosis of hydatidiform moles. In turn, the early detection and management have de-emphasised histopathological characteristics that were once regarded as diagnostic evidence by making them less typical and more delicate. In addition, interobserver and intraobserver variability remain as limitations to morphological diagnosis and the histopathologic criteria have failed to keep up and offer a satisfactory diagnosis. For this reason, many novel detection methods have been introduced to narrow the gap, 


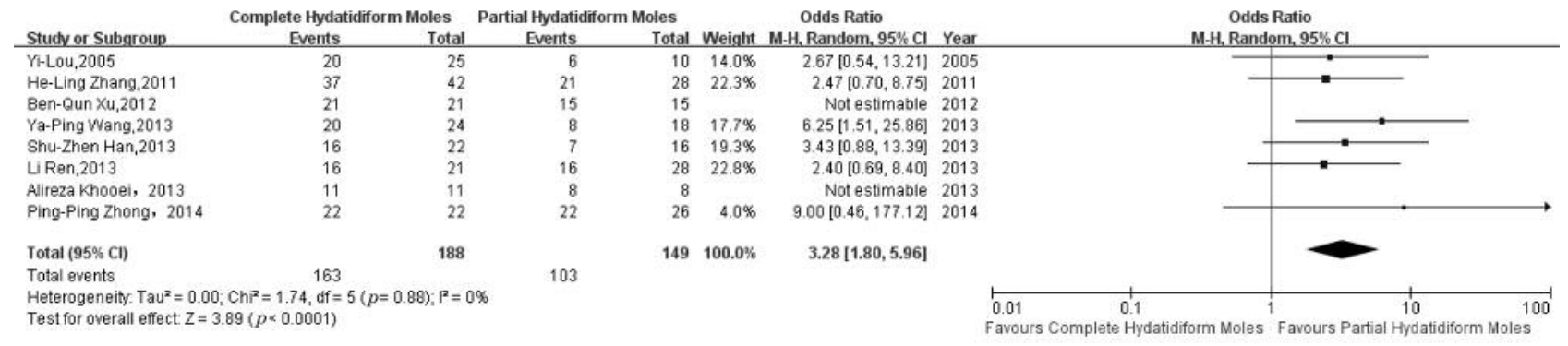

Figure 2. Effect of overall positive rate of Ki-67 in discriminating between complete and partial hydatidiform moles.

Table II. Characteristics of the eight identified studies that met the predefined inclusion criteria.

\begin{tabular}{|c|c|c|c|c|c|c|c|c|c|c|c|c|}
\hline \multirow[t]{2}{*}{ Study } & \multirow[t]{2}{*}{ Country } & \multirow[t]{2}{*}{ Design } & \multicolumn{5}{|c|}{ Partial hydatidiform moles } & \multicolumn{5}{|c|}{ Complete hydatidiform moles } \\
\hline & & & $(-)$ & $(+)$ & $(++)$ & $(+++)$ & $\begin{array}{c}\text { No. of } \\
\text { participants }\end{array}$ & $(-)$ & $(+)$ & $(++)$ & $(+++)$ & $\begin{array}{c}\text { No. of } \\
\text { participants }\end{array}$ \\
\hline Yi-Lou, 2005 & China & NRCT & 4 & 6 & 0 & 0 & 10 & 5 & 18 & 2 & 0 & 25 \\
\hline He-Ling Zhang, 2011 & China & NRCT & 7 & 15 & 6 & 0 & 28 & 5 & 23 & 9 & 5 & 42 \\
\hline Ben-Qun Xu, 2012 & China & NRCT & 0 & 14 & 1 & 0 & 15 & 0 & 2 & 19 & 0 & 21 \\
\hline Alireza Khooe, 2013 & Iran & NRCT & 0 & 3 & 5 & 0 & 8 & 0 & 0 & 5 & 6 & 11 \\
\hline Shu-Zhen Han, 2013 & China & NRCT & 9 & \multicolumn{3}{|c|}{7} & 16 & 6 & & \multicolumn{2}{|c|}{16} & 22 \\
\hline Ya-Ping Wang, 2013 & China & NRCT & 10 & 5 & 3 & 0 & 18 & 4 & 6 & 6 & 8 & 24 \\
\hline Li Ren, 2013 & China & NRCT & 12 & 6 & 6 & 4 & 28 & 5 & 2 & 6 & 8 & 21 \\
\hline Ping-Ping Zhong, 2014 & China & NRCT & 4 & \multicolumn{3}{|c|}{22} & 26 & 0 & 0 & 0 & 22 & 22 \\
\hline
\end{tabular}

including DNA ploidy analysis, short-tandem-repeat (STR) genotyping and chromosomal enumeration by fluorescent in situ hybridisation (FISH). However, most of these techniques are based at the genetic level and tend to be too timeconsuming, inconvenient and economically inefficient for routine use in clinical practice.

The similarities in the characteristics of healthy trophoblast and malignant cells - invasiveness, high cell proliferation, lack of cell contact inhibition and immune exemption - prompted us to attempt to apply biomarkers of malignant cells for identification of hydatidiform moles. Ki67 is one of these biomarkers (31-35).

Quantitative assessment of immunoreactivity of the Ki-67 antigen (MIB1) through the use of immunohistochemical methods has been established as a simple alternative to more complex genetic assessment techniques. By eliminating dependence on expensive or sophisticated equipment and by virtue of its flexibility in the tissues that can be used, assessment of immunoreactivity of Ki-67 with immunohistochemical methods can complement gene level techniques such as deoxyribonucleic acid ploidy analysis, which are mainly used for research rather than clinical purposes.

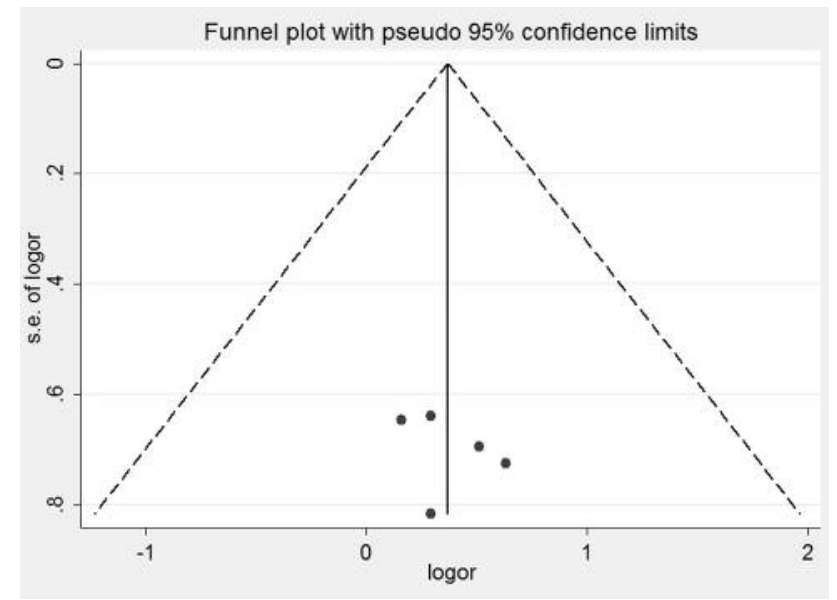

Figure 3. Funnel plots of overall positive rate of Ki-67 in complete and partial hydatidiform moles. s.e: Standard error; or: odds ratio.

However, the diagnostic value of Ki-67 analysis in discriminating between partial and complete hydatidiform moles and making a precise subdivision between the two 
types is still an issue with many unanswered questions and significant controversy.

Overall analysis. This systematic review and meta-analysis included eight trials with a total of 337 participants who met the inclusion criteria, and revealed higher Ki-67 expression in complete than in partial hydatidiform moles and the difference was statistically significant. We assessed the methodological quality of these eligible studies by using the NOS approach. All studies clearly described the patient characteristics in the selection criteria and obtained the full score (nine stars) according to the NOS, except for one study that got eight stars. Details of procedures such as patient preparation, material drawings, previous tissue treatment, gestational age and mean age of participants were reported in detail. The reasonable designs and high levels of evidence upgraded the quality of our analysis.

Quantitative assessment of immunoreactivity of Ki-67 through immunohistochemical methods, coupled with histopathologic studies, may improve the accuracy of subdividing hydatidiform moles into partial and complete types and seems to be a fairly rapid, simple, economical and, above all, efficient technique. Its clinical value deserves attention. However, the novel detection method will not substitute histopathologic studies as the gold standard, and it will only be a supplement and help with early diagnosis and improve diagnostic accuracy. In future, large-scale, highquality and multi-institutional trials should also be conducted to reveal the role of this promising biomarker in the precise and early diagnosis of hydatidiform moles.

Comparison with other reviews. Our review was based on systematic literature searches and strict inclusion criteria and included assessments of the methodological quality and risk of bias in the studies that met the predefined inclusion criteria using the NOS approach and the PRISMA guidelines. To our knowledge, this study is the first meta-analysis to compare complete and partial hydatidiform moles for the purpose of exploring the potential value of $\mathrm{Ki}-67$ as a marker for differential diagnosis between subgroups of hydatidiform moles. Our review differs from the previously published work as it contrasts and combines results from different studies, thereby increasing statistical power (over individual studies) with information aggregation, and resolving uncertainty when reports disagree. In the present work, patterns and sources of inconsistency among different study results were quantified and analyzed. The current results can, therefore, be generalized to a larger population, and the precision and accuracy of estimates can be improved as more data are used. This, in turn, may increase the statistical power to detect an effect.

Study limitations. Limitations of our study relate to the quality and quantity of the available evidence on this issue. First, the sources of publications searched from selected electronic databases were limited, and we failed to retrieve unpublished studies, thus potentially introducing inevitable publication bias. Second, due to race and geographical variations of incidence, even though our search strategy disregarded language limitations, we failed to find all qualified studies in other languages, which may produce language bias. Moreover, age, family history, and other covariates that may contribute to the incidence of the disease were also not considered.

\section{Conflicts of Interest}

The Authors report no conflict of interest.

\section{Acknowledgements}

The Authors are grateful to Prof. Yiming Zhao (Peking University, China) for providing important guidance related to statistical methodology. Th Authors also would like to thank Prof. Xiao Han (Nanjing Medical University) and Prof. Yongqing Wang (Peking University Third Hospital, China) for their expertise. The Authors gratitude is also extended to Prof. Dongmei Tian (Peking University) for providing suggestions and correcting grammatical and syntax errors.

\section{References}

1 Wells M: The pathology of gestational trophoblastic disease: recent advances. Pathology 39: 88-96, 2007.

2 Szulman AE: Trophoblastic disease: clinical pathology of hydatidiform moles. Obstet Gynecol Clin North Am 15: 443456, 1998.

3 Berkowitz RS and Goldstein DP: Clinical practice. Molar pregnancy. N Engl J Med 360: 1849-1645, 2009.

4 Feltmate CM, Growdon WB and Wolfberg AJ: Clinical characteristics of persistent gestational trophoblastic neoplasia after partial hydatidiform molar pregnancy. J Reprod Med 51: 902-906, 2006.

5 Hui P, Martel $\mathrm{M}$ and Parkash V: Gestational trophoblastic diseases: recent advances in histopathologic diagnosis and related genetic aspects. Adv Anat Pathol 12: 116-125, 2005.

6 Sebire NJ, Fisher RA and Rees HC: Histopathological diagnosis of partial and complete hydatidiform mole in the first trimester of pregnancy. Pediatr Dev Pathol 6: 69-77, 2003.

7 Howat AJ, Beck S, Fox H, Harris SC, Hill AS, Nicholson CM and Williams RA: Can histopathologists reliably diagnose molar pregnancy? J Clin Pathol 46: 599-602, 1993.

8 Messerli ML, Parmley T, Woodruff JD, Lilienfeld AM, Bevilacqua L and Rosenshein NB: Inter and intra-pathologist variability in the diagnosis of gestational trophoblastic neoplasia. Obstet Gynecol 69: 622-626, 1987.

9 Buza $\mathrm{N}$ and Hui P: Partial hydatidiform mole: histologic parameters in correlation with DNA genotyping. Int J Gynecol Pathol 32: 307-315, 2013.

10 Gupta M, Vang R, YemelyanovaAV, Kurman RJ, Li FR, Maambo EC, Murphy KM, DeScipio C, Thompson CB and Ronnett BM: Diagnostic reproducibility of hydatidiform moles: ancillary techniques (p57) immunohistochemistry and molecular 
genotyping) improve morphologic diagnosis for both recently trained and experienced gynecologic pathologists. Am J Surg Pathol 36: 1747-1760, 2012.

11 Genest DR: Partial hydatidiform mole: clinicopathological features differential diagnosis, ploidy and molecular studies, and gold standards for diagnosis. Int J Gynecol Pathol 20: 315-322, 2001.

12 Maggiori MS and Peres LC: Morphological, immunohistochemical and chromosome in situ hybridization in the differential diagnosis of Hydatidiform Mole and Hydropic Abortion. Eur J Obstet Gynecol Reprod Biol 135: 170-176, 2007.

13 Hui P: Molecular diagnosis of gestational trophoblastic disease. Expert Rev Mol Diagn 10: 1023-1264, 2010.

14 Kajii $\mathrm{T}$ and Ohama K: Androgenetic origin of hydatidiform mole. Nature 268: 633-634, 1977.

15 Schammel DP and Bocklage T: P53, PCNA, and Ki-67 in hydropic molar and nonmolar placentas: an immunohistochemical study. Int J Gynecol Pathol 15: 158-166, 1996.

16 Ostrzega N, Phillipson J and Liu P: Proliferative activity in placentas with hydropic change and hydatidiform mole as detected by Ki-67 and proliferating cell nuclear antigen immunostaining. Am J Clin Pathol 110: 776-781, 1998.

17 Cheung ANY, Ngan HY, Chen WZ, Loke SL and Collins RJ: The significance of proliferating cell nuclear antigen in human trophoblastic disease: an immunohistochemical study. Histopathology 22: 565-568, 1993.

18 Cheung AN, Ngan HY, Collins RJ and Wong YL: Assessment of cell proliferation in hydatidiform mole using monoclonal antibody MIB1 to Ki-67 antigen. J Clin Pathol 47: 601-604, 1994.

19 Suresh UR, Hale RJ, Fox H and Buckley CH: Use of proliferation cell nuclear antigen immunoreactivity for distinguishing hydropic abortions from partial hydatidiform moles. J Clin Pathol 46: 48-50, 1993.

20 Jeffers MD, Grehan D and Gillan JE: Comparison of villous trophoblast proliferation rate in hydatidiform mole and nonmolar abortion by assessment of proliferating cell nuclear antigen expression. Placenta 15: 551-556, 1994.

21 Whiting P, Rutjes AW, Reitsma JB, Bossuyt PM and Kleijnen J: The development of THE NEWCASTLE-OTTAWA SCALE (NOS): a tool for the quality assessment of studies of diagnostic accuracy included in systematic reviews. BMC Med Res Methodol 3: 25, 2003.

22 Moher D, Liberati A, Tetzlaff J, Altman DG and PRISMA Group: Preferred reporting items for systematic reviews and meta-analyses: the PRISMA statement. Int J Surg 8: 336-341, 2010.

23 Lou Yi, Cai Hong-Guang, Shi Xiao-Liang, Ruan Xiu-Lan and $\mathrm{Fu} \mathrm{Zi}-\mathrm{Mu}$ : Expression of PCNA and Ki-67 in hydatidiform moles in different sites of uterine cavity and their clinical values. Chinese J Birth Health Hered 13(12): 33-35, 2005.

24 Zhang He-Ling, Xu Huan-Zhi and Wang Su-Qin: The expression and clinical Value of Ki-67 in different hydatidiform mole tissues. Hebei Med J 33(11): 1697-1698, 2011.
25 Xu Ben-Qun, Zhou Qiong, Xing Yi-Xiang and Chen Chong: Expression and clinical significance of CD146 and Ki67 in diagnosis of hydatidiform mole. China Med Pharm 2(17): 29-31, 2012.

26 Khooei A, Atabaki Pasdar F, Fazel A, Mahmoudi M, Nikravesh MR, Khaje Delui M and Pourheydar B: Ki-67 expression in hydatidiform moles and hydropic abortions. Iran Red Crescent Med J 15(7): 590-594, 2013.

27 Han Shu-Zhen, Guo Ying, Huang Bin-Hua, Zhao Ying-Hai and Chen Xiao-Yi: The Utility of p57Kip2, Ki-67 in differential diagnosis of hydatidiform mole and hydropic abortion in early pregnancy stage. China Modern Doctor 51(25): 140-141, 2013.

28 Wang Ya-Ping, Liu Ling, Yu Jing-Gong, Li Guang-Hui, Song Guang-Yao and Zou Na: The expression and clinical Value of p53 and Ki-67 in hydatidiform moles. China Prac Med 8(11): 246-247, 2013.

29 Ren Li, Hou Zhao-Hui, Guo Xiao-Dong, Zheng Ji-Chun, Li DeChang, Yue Ying, ZHU Jian-Ping and Liu Ming: Significance on the Combination of p57, p53, bcl-2, ki-67 Applied to the Diagnosis on Hydatidiform Mole. Progress Modern Biomed 13(7): 1319-1322, 2013.

30 Zhong Ping-Ping, Gu Yi-Qun, Sun Yun-Fei, Lu Li-Juan and Wang Ai-Chun: Diagnostic value of p57, p53 and Ki-67 protein in hydatidiform mole. J Diag Pathol 21(6): 391-394, 2014.

31 Chen Y, Shen D, Gu Y, Zhong P, Xie J and Song Q: The diagnostic value of Ki-67, P53 and P63 in distinguishing partial Hydatidiform mole from hydropic abortion. Wien Klin Wochenschr 124(5-6): 184-187, 2012.

32 Kale A, Söylemez F and Ensari A: Expressions of proliferation markers (Ki-67, proliferating cell nuclear antigen, and silverstaining nucleolar organizer regions) and of p53 tumor protein in gestational trophoblastic disease. Am J Obstet Gynecol 184(4): 567-574, 2001.

33 Hasanzadeh M, Sharifi N, Esmaieli H, Daloee MS and Tabari A: Immunohistochemical expression of the proliferative marker Ki67 in hydatidiform moles and its diagnostic value in the progression to gestational trophoblastic neoplasia. J Obstet Gynaecol Res 39(2): 572-577, 2013.

34 Xue WC, Khoo US, Ngan HY, Chan KY, Chiu PM, Tsao SW and Cheung AN: Minichromosome maintenance protein 7 expression in gestational trophoblastic disease: correlation with Ki67, PCNA and clinicopathological parameters. Histopathology 43(5): 485-490, 2003.

35 Makovitzky J, Radtke A, Shabani N, Friese K, Gerber B and Mylonas I: Invasive hydatidiform mole: immunohistochemical labelling of inhibin/activin subunits, Ki67, p53 and glycodelin A in a rare case. Acta Histochem 111(4): 360-365, 2009.

Received November 9, 2017

Revised November 24, 2017 Accepted November 27, 2017 